\title{
The role of serum D-dimer in the diagnosis of periprosthetic joint infection and timing of reimplantation
}

\author{
Erdem Sahin, Bedri Karaismailoglu, Mahmut Kursat Ozsahin, Huseyin Botanlioglu, Gokhan Kaynak
}

From the Erzurum Regional Training and Research Hospital, Erzurum, Turkey

D-dimer is a fibrin degradation product formed by the destruction of the cross-linked fibrin clot by activation of the coagulation system. Many studies have shown that systemic and local infections cause fibrinolytic activities. The purpose of this study was to determine the role of serum D-dimer in the diagnosis of periprosthetic joint infection (PJI) and the timing of reimplantation. The patients who underwent primary and revision knee or hip arthroplasty between July 2018 and May 2019 were prospectively evaluated. All surgeries were performed by the same surgeon. 60 of 71 patients who met our criteria were included in the study, comprising of 27 primary arthroplasties (PA), 21 two-stage septic revision (SR) and 12 aseptic revision (AR). We analyzed D-dimer, C-reactive protein (CRP) and erythrocyte sedimentation rate (ESR) levels. The values of the septic group were evaluated as two groups; Group 1 (before the first stage) and Group 2 (before the second stage) to investigate the role of D-dimer in determining the timing of reimplantation. D-dimer level was significantly higher in the SR group compared to $P A$ and AR groups $(p<0.001$ and $p=0.045)$. CRP and ESR levels were significantly higher in the SR group compared to PA and AR groups. There was no statistically significant difference between preoperative D-dimer levels of Group 1 and Group 2

Source of funding: this research did not receive any specific grant from funding agencies in the public, commercial, or not-for-profit sectors.

Compliance with ethical standards: all procedures performed in the studies involving human participants were in accordance with the ethical standards of the institutional and/or national research committee and with the 1964 Declaration of Helsinki and its later amendments or comparable ethical standards. Written and signed informed consent was obtained from all patients. $(p=0.2)$. Serum $D$-dimer level can be used as an easy and inexpensive test to support the diagnosis of PJI. But the D-dimer level is not useful to determine the timing of reimplantation.

Keywords: periprosthetic joint infection; diagnosis; arthroplasty; knee revision; hip revision; d-dimer.

\section{INTRODUCTION}

The number of periprosthetic joint infections (PJI) is increasing day by day, due to the more common application of the primary joint arthroplasty. Diagnosis and treatment are challenging for arthroplasty surgeons. There is no single absolute diagnostic test, therefore the clinicians try to confirm the diagnosis by using a combination of synovial

\footnotetext{
Erdem Sahin ${ }^{1}$

Bedri Karaismailoglu²

- Mahmut Kursat Ozsahin ${ }^{2}$

Huseyin Botanlioglu²

- Gokhan Kaynak ${ }^{2}$

${ }^{1}$ Erzurum Regional Training and Research Hospital, Erzurum, Turkey.

${ }^{2}$ Istanbul University-Cerrahpasa, Cerrahpasa Medical Faculty, Department of Orthopaedics and Traumatology, Istanbul, Turkey.

Correspondence : Gokhan Kaynak, Cerrahpasa Mahallesi Kocamustafapasa Caddesi No:34/E, Fatih/Istanbul, Turkey. Phone: +90 532 4563536, Fax: +90 2124143000.

Email : kaynak95@hotmail.com

- 2021, Acta Orthopædica Belgica.
} 
fluid tests and serological markers. Musculoskeletal Infection Society (MSIS) developed an algorithm for the diagnosis of PJI and these criteria were updated in 2018 by the International Consensus Meeting (ICM) on Periprosthetic Joint Infection (1). Although many serological markers have been evaluated for the diagnosis of PJI, the most widely used tests are Erythrocyte Sedimentation Rate (ESR) and C-Reactive Protein (CRP) levels. Although the synovial fluid biomarkers are invaluable for the diagnosis of PJI, they can remain inconclusive due to the inadequate volume of the sample, furthermore; the use of some markers such as alpha defensin is expensive.

The timing for reimplantation is also controversial. Although ESR and CRP levels are reliable tests for the diagnosis of PJI, their use to decide the optimal timing of implantation is limited since they can remain high in the postoperative period $(2,3)$. Two different studies demonstrated that ESR and CRP levels during reimplantation did not predict the outcome of the treatment $(4,5)$. $\mathrm{D}$-dimer is a fibrin degradation product formed by the destruction of the cross-linked fibrin clot by activation of the coagulation system. Many studies have shown that systemic and local infections cause fibrinolytic activities. In a recent study by Parvizi et al., D-dimer was found to be a valuable marker for both the diagnosis and reimplantation time in PJI. [9] D-dimer was also included as a minor criterion in the latest version of diagnostic criteria published in 2018 (1). In light of the current literature, we hypothesized that serum D-dimer is a valuable marker both for the diagnosis PJI and it can also help to show the optimal timing of reimplantation.

\section{MATERIALS AND METHODS}

This study was performed prospectively on patients who underwent primary and revision knee and hip arthroplasty between July 2018 and May 2019. It was approved by the institutional ethics review board. The demographic data of the patient including sex, age, the involved joint, isolated organisms, and comorbidities were recorded.

Patients were divided into three groups; 38 patients undergoing total knee and total hip arthro-

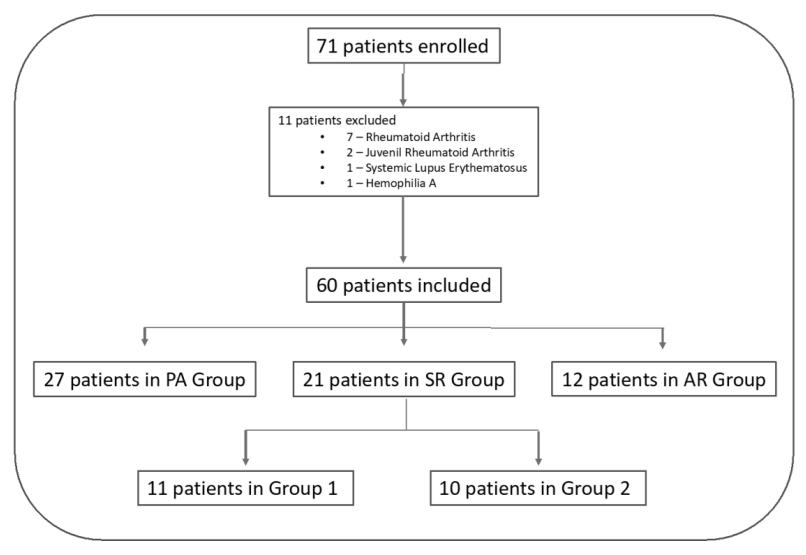

Figure 1. - Study enrollment schema.

plasty for primary gonarthrosis or coxarthrosis (PA group), 21 patients undergoing two-stage septic revision arthroplasty due to periprosthetic infection (SR group) and 12 patients undergoing revision arthroplasty due to aseptic failure (AR group) (Figure 1). We also divided the SR group into two groups as the preoperative period of the first stage; spacer application (Group 1) and second stage (reimplantation) (Group 2) to evaluate the role of D-dimer levels for the reimplantation time. There were 11 patients in Group 1 and 10 patients in Group 2 . None of the patients had a concomitant infection. We excluded the 11 patients who had Rheumatoid Arthritis (RA) or other systemic inflammatory diseases in the primary arthroplasty group because these conditions can affect the D-dimer level (6). ESR and CRP levels were measured in the venous blood samples taken when the patients consulted at our clinic. D-dimer levels were measured in the blood samples taken in preoperative anesthesia preparation. ESR levels ( $\mathrm{mm} / \mathrm{hr}$ ), CRP levels ( $\mathrm{mg} / \mathrm{l})$ and D-dimer levels $(\mu \mathrm{g} / \mathrm{ml})$ were obtained by the Westergren method, Turbidimetric method and KOA method, respectively. The patients undergoing revision surgery were categorized into septic and aseptic according to the finalized ICM criteria in 2013 (7).

A two-stage revision was used in all PJIs. After the first stage, patients stayed under antibiotherapy for 6 weeks and then the reimplantation was done if normal or down-trending CRP and ESR values were obtained. Culture specimens were obtained 
Table 1. - The demographic characteristics of the patients

\begin{tabular}{|l|c|c|c|c|}
\hline \multicolumn{1}{|l|}{ PA GROUP } & SR GROUP & AR GROUP & p value \\
\hline Age & $56.81 \pm 12.23$ & $64.19 \pm 8.20$ & $57.08 \pm 7.62$ & $0.036^{*}$ \\
\hline Gender & $6(42.9 \%)$ & $5(35.7 \%)$ & $3(21.4 \%)$ & \multirow{2}{*}{$1 * *$} \\
\hline Male & $21(45.7 \%)$ & $16(34.8 \%)$ & $9(19.6 \%)$ & \\
\hline Female &
\end{tabular}

*Post hoc comparisons of age: PA-SR: $\mathrm{p}=0.046$, PA-AR: $\mathrm{p}=1$, SR-AR: $\mathrm{p}=0.174$. **One Way Anova and Fisher Freeman Halton tests.

Table 2. - Comparison of the parameters between the study groups

\begin{tabular}{|c|c|c|c|c|c|}
\hline $\begin{array}{l}\text { Preoperative } \\
\text { Levels }\end{array}$ & GROUP PA & GROUP SR & GROUP AR & p value* & Post-hoc p value** \\
\hline $\begin{array}{c}\text { D-dimer }(\boldsymbol{\mu g} / \mathbf{m l}) \\
\text { Median } \\
\text { Mean }\end{array}$ & $\begin{array}{c}0.62(0.19-1.96) \\
0.47( \pm 0.18)\end{array}$ & $\begin{array}{c}2.94(0.24-7.73) \\
3.01( \pm 1.76)\end{array}$ & $\begin{array}{c}1.14(0.34-4.65) \\
1.5( \pm 1.34)\end{array}$ & $\mathrm{p}<0.001$ & $\begin{array}{l}\text { PA-SR: } p<0.001 \\
\text { SR-AR: } p=0.045\end{array}$ \\
\hline $\begin{array}{c}\text { CRP }(\mathbf{m g} / \mathbf{L}) \\
\text { Median } \\
\text { Mean }\end{array}$ & $\begin{array}{c}3.77(0.55-11.73) \\
\quad 4.15( \pm 3,07)\end{array}$ & $\begin{array}{c}15.45(2.03-241) \\
33( \pm 53.5)\end{array}$ & $\begin{array}{c}3.86(1.28-23.12) \\
6.7( \pm 7.2)\end{array}$ & $p=0.001$ & $\begin{array}{l}\text { PA-SR: } p<0.001 \\
\text { SR-AR: } p=0.036\end{array}$ \\
\hline $\begin{array}{c}\text { ESR }(\mathbf{m m} / \mathbf{h r}) \\
\text { Median } \\
\text { Mean }\end{array}$ & $\begin{array}{c}18(1-42) \\
13.77( \pm 8.02)\end{array}$ & $\begin{array}{c}50(7-91) \\
50.3( \pm 24.2)\end{array}$ & $\begin{array}{c}21(2-32) \\
20.3( \pm 13.8)\end{array}$ & $\mathrm{p}<0.001$ & $\begin{array}{l}\text { PA-SR: } p<0.001 \\
\text { SR-AR: } p=0.001\end{array}$ \\
\hline
\end{tabular}

*Kruskal Wallis test. **Dunn test.

from tissue samples and joint fluid taken during revision surgery. Tissue samples were taken with sharp dissection. The synovial fluid was taken by aspiration before the joint capsule was opened. The samples were collected in three to six pediatric blood culture bottles and were sent to the laboratory immediately. The cultures were incubated for up to 14 days.

The distribution of the data was examined by the Shapiro Wilk test. One way Anova test was used for comparison of three or more groups with a normal distribution. Kruskal Wallis test was used to compare three or more groups if they are not normally distributed. Descriptive statistics of the data are given as $\mathrm{n}(\%)$, median (min-max) and mean \pm standard deviation. Bonferroni and Dunn tests were used as post hoc test. All statistical analyses were performed using IBM SPSS Statistics 22.0 program and $\mathrm{p}$ value less than 0.05 was considered significant.

\section{RESULTS}

Sixty patients were included in the final analysis. The mean age of patients was $59( \pm 10) .14$ patients $(\% 23.3)$ were male and 46 patients $(\% 76.7)$ were female. It was observed that the groups were homogeneous for gender $(\mathrm{p}=1)$, but not homogenous for age $(\mathrm{p}=0.036)($ Table 1$)$.

There was a statistically significant difference between the groups in terms of the preoperative D-dimer levels $(p<0.001)$. Post-hoc analyses showed that there was a significant difference between Group PA-SR and Group SR-AR. The average D-dimer level in Group SR was significantly higher than Group PA and AR. There was a statistically significant difference between the groups in terms of the preoperative CRP levels $(\mathrm{p}=0.001)$. Posthoc analyses showed that there was a significant difference between Groups PA-SR and Groups SR-AR. The average CRP level in Group SR was 
Table 3. - Comparison of D-dimer levels of the patients in Group 1 and Group 2

\begin{tabular}{|c|c|c|c|}
\hline $\begin{array}{c}\text { Preoperative } \\
\text { Levels }\end{array}$ & GROUP 1 & GROUP 2 & $\begin{array}{c}\text { p } \\
\text { value* }^{*}\end{array}$ \\
\hline $\begin{array}{c}\text { D-dimer } \\
\text { Median } \\
\text { Mean }\end{array}$ & $\begin{array}{c}2.59(0.48-7.73) \\
2.64( \pm 1.91)\end{array}$ & $\begin{array}{c}3.73(0.24-5.34) \\
3.43( \pm 1.57)\end{array}$ & 0.2 \\
\hline
\end{tabular}

*Kruskal Wallis test.

Table 4. - Correlation between D-dimer and CRP levels of the patients in Group SR (septic revision)

\begin{tabular}{|c|c|c|c|}
\hline & & & Preop CRP \\
\hline Spearman's rho & Preop D-dimer & $\mathbf{r}^{*}$ & -0.364 \\
\hline & & p-value & 0.105 \\
\hline
\end{tabular}

*r: correlation coefficient.

significantly higher than in Groups PA and AR. There was a statistically significant difference between the groups in terms of the preoperative ESR levels $(p<0.001)$. Post-hoc analyses showed that there was a significant difference between Groups PA-SR and Group SR-AR. The average ESR level in Group SR was significantly higher than in Groups PA and AR. (Table 2) There was no statistically significant difference between the average D-dimer levels of the patients in Group 1 and Group $2(\mathrm{p}=0.2)$ (Table 3 ). There was no statistically significant correlation between D-dimer and CRP levels of the patients in Group SR ( $\mathrm{r}=0.364, \mathrm{p}=0.105)$ (Table 4).

\section{DISCUSSION}

The results of this study suggest that D-dimer is a useful marker for the diagnosis of PJI. However, it is not helpful for determining the timing of reimplantation.

Septic loosening after total joint arthroplasty occurs in $1-4 \%$ of the patients (8). Aseptic loosening should be distinguished from septic loosening since they require different treatment procedures. However, it is challenging in most cases. Recently, synovial fluid biomarkers are shown to be useful in diagnosing PJI. Combined analysis of the alphadefensin and CRP in synovial fluid for the diagnosis of PJI showed $97 \%$ sensitivity and $100 \%$ specificity (9). The fact that the alpha-defensin test is expensive has led clinicians to investigate different markers to aid in the differential diagnosis. Numerous different markers are suggested and being analyzed in the literature. D-dimer is also one of them and has aroused interest due to its easy and cheap application.

Ribera et al. showed that D-dimer concentration in synovial fluid is increased 15 times in foals with a septic joint disease. The study confirmed the opinion that D-dimer is associated with inflammation or infection in the joint. The products of fibrinolytic activity such as D-dimer pass into the systemic circulation and thus it can be measured in venous blood (10). In a prospective study which included primary and revision arthroplasty patients, serum D-dimer outperformed both ESR and serum CRP with $89.5 \%$ sensitivity and $92.8 \%$ specificity, suggesting an optimal serum D-dimer cut-off value of $850 \mathrm{ng} / \mathrm{ml}$ for the diagnosis of PJI (9).

In our study, preoperative D-dimer levels in the SR group were found to be significantly higher compared to the patients in PA and AR groups. In the SR group, D-dimer was measured before the first stage of spacer insertion in 11 patients and before the second stage of reimplantation in 10 patients. The results of perioperative cultures were positive in 5 of 10 patients who were evaluated with D-dimer before the reimplantation. In patients who had positive culture results, CRP and ESR values were normal before reimplantation. However, D-dimer levels were high in both culture-positive and negative patients. When D-dimer levels before the first and second stage were compared, there was no statistically significant difference $(p=0.2)$.

In the study of Lee et al., D-dimer levels increased sharply and peaked on the first postoperative day (the highest level was $4.5 \mu \mathrm{g} / \mathrm{ml}$ ), then it almost regressed to the initial level on the second postoperative day and then gradually increased again and reached the second peak at the second postoperative week. It almost decreased to the normal level in the sixth postoperative week (11). In our study, only one of the septic patients had a normal D-dimer value at 6 week, while the D-dimer values of the other nine patients remained 
high until the 8 th week or later. Reinfection did not occur in any of the patients who had a positive or negative perioperative culture at minimum 6 months follow-up. Due to the fact that the D-dimer level of septic patients before the second stage of reimplantation was as high as before the first stage (spacer application), the D-dimer level had limited guiding for reimplantation time. However, Shahi et al. reported that an elevated D-dimer before reimplantation could be an indication of persistent infection (9).

There was no statistically significant correlation between the D-dimer and CRP levels of the patients in SR group. It means that the increases and decreases in D-dimer and CRP levels were not parallel, thus the elevation of these parameters can correspond to different stages of the PJI. Therefore, the D-dimer and CRP levels should be used as a separate parameter in the diagnosis of PJI. In our study, serum CRP and ESR levels were higher in the infected group, similar to the literature (5). We also found higher D-dimer levels in SR group than in AS and PA groups. This data shows us that serum D-dimer level can be used as the diagnostic marker of PJIs in accordance with Shahi et al.'s study (9).

Although D-dimer was found as a useful marker for PJI diagnosis, it was concluded that the D-dimer levels have no guiding effect in determining the reimplantation time in patients who underwent twostage revision arthroplasty due to PJI, since there was no statistically significant difference between preoperative D-dimer values of 11 patients who underwent spacer application and 10 patients who underwent reimplantation.

Although it is a prospective study, our study has several limitations. Firstly, the patient number was low compared to some available literature. D-dimer has a low specificity and high D-dimer concentrations can also be observed in patients with increased fibrin production, such as cancer, infection, inflammation, surgery, injuries, hemorrhages and other clinical conditions (9). Some additional undiagnosed problems of the patients might have led to an increased concentration of D-dimer. Finally, although D-dimer values were found to be significantly different in the infected group, threshold value, sensitivity, and specificity analyses were not performed, due to the small sample size leading to non-significant results.

\section{CONCLUSION}

Serum D-dimer measurement is easily accessible and can be an effective diagnostic test for early detection of PJI. However, we need further validation studies to support the findings and confirm the relative test performance of D-dimer compared to other commonly used serum markers. The D-dimer was not found successful in determining the timing of the reimplantation.

\section{REFERENCES}

1. Parvizi J, Tan TL, Goswami K, Higuera C, Della Valle C, Chen AF, et al. The 2018 Definition of Periprosthetic Hip and Knee Infection: An Evidence-Based and Validated Criteria. J Arthroplasty. 2018 May; 33(5): 1309-1314.e2.

2. Larsson S, Thelander U, Friberg S. C-reactive protein (CRP) levels after elective orthopedic surgery. Clin Orthop Relat Res. 1992; (275): 237-42.

3. Bilgen Ö, Atici T, Durak K, Karaeminoğullari O, Bilgen M. C-Reactive Protein Values and Erythrocyte Sedimentation Rates after Total Hip and Total Knee Arthroplasty. J Int Med Res. 2001 Feb 25; 29(1): 7-12.

4. Kusuma SK, Ward J, Jacofsky M, Sporer SM, Della Valle CJ. What is the role of serological testing between stages of two-stage reconstruction of the infected prosthetic knee? Clin Orthop Relat Res. 2011; 469(4): 1002-8.

5. Ghanem E, Antoci V, Pulido L, Joshi A, Hozack W, Parvizi J. The use of receiver operating characteristics analysis in determining erythrocyte sedimentation rate and C-reactive protein levels in diagnosing periprosthetic infection prior to revision total hip arthroplasty. Int J Infect Dis. 2009; 13(6).

6. Busso N, Hamilton JA. Extravascular coagulation and the plasminogen activator/plasmin system in rheumatoid arthritis. Arthritis Rheum. 2002; 46(9): 2268-79.

7. Cats-Baril W, Gehrke T, Huff K, Kendoff D, Maltenfort M, Parvizi J. International consensus on periprosthetic joint infection: Description of the consensus process. Clin Orthop Relat Res. 2013; 471(12): 4065-75.

8. Love C, Tomas MB, Marwin SE, Pugliese P V., Palestro CJ. Role of nuclear medicine in diagnosis of the infected joint replacement. Radiographics. 2001; 21(5): 1229-38.

9. Shahi A, Kheir MM, Tarabichi M, Hosseinzadeh HRS, Tan TL, Parvizi J. Serum D-Dimer Test Is Promising for the Diagnosis of Periprosthetic Joint Infection and Timing of Reimplantation. J Bone Jt Surg - Am Vol. 2017; 99(17): 1419-27. 
10. Ribera T, Monreal L, Armengou L, Ríos J, Prades M. Synovial Fluid D-Dimer Concentration in Foals with Septic Joint Disease. J Vet Intern Med. 2011; 25(5): 1113-7.
11. Lee YS, Lee YK, Han SB, Nam CH, Parvizi J, Koo KH. Natural progress of D-dimer following total joint arthroplasty: A baseline for the diagnosis of the early postoperative infection. J Orthop Surg Res. 2018; 13(1). 